\title{
Effects of endurance exercise on serum inflammatory cytokine level and kidney structure in a rat diabetes model
}

\author{
SEN LIN，SHANHU JIN，FENGLIN ZHOU，YAZHE HU and MING ZHANG \\ Department of Sport Science, School of Physical Education, Central China Normal University, \\ Wuhan, Hubei 430070, P.R. China
}

Received January 4, 2020; Accepted December 8, 2020

DOI: $10.3892 / \mathrm{etm} .2021 .10559$

\begin{abstract}
Diabete mellitus (DM) is becoming a global health problem. Whilst many studies have previously focused on the therapeutic effects of exercise on diabetes, insufficient data exist on its effectiveness on disease prevention. The present study was designed to evaluate the effects of endurance exercises on kidney injury and on the expression of metalloproteinases (MMPs), tissue inhibitors of metalloproteinases (TIMPs) and transforming growth factor (TGF)- $\beta 1$. Type 2 diabetic rat model was created followng 8 weeks of feeding on high fat diet, followed by injection with streptozotocin $(\mathrm{STZ} ; 30 \mathrm{mg} / \mathrm{kg}$ ). A total of three different intensity endurance exercises, including low-intensity exercise $(8 \mathrm{~m} / \mathrm{min}$ and $0^{\circ}$ slope), moderate-intensity exercise $\left(15 \mathrm{~m} / \mathrm{min}\right.$ and $5^{\circ}$ slope $)$ and high-intensity exercise $\left(20 \mathrm{~m} / \mathrm{min}\right.$ and $10^{\circ}$ slope $)$, were arranged during this process. Oral glucose tolerance (OGTT) and oral sucrose tolerance tests (OSTT) were performed in all rats 1 week after STZ injection. Serum interleukin-6 and tumor necrosis factor- $\alpha$ levels were measured using ELISA. After OGTT, all rats were sacrificed and kidney samples were removed for hematoxylin and eosin staining and western blot analyzes. Urea and ureatinine levels, representative of renal function, were estimated by using automatic biochemical analyzer. Rats in the DM group showed severe impaired glucose tolerance, which was alleviated in the moderate-intensity exercise (ME) and the high-intensity exercise (HE) groups. Inflammatory cytokines were also significantly reduced rats in the ME group compared with those in the DM group. No difference in renal function, MMP-9/TIMP-1 and TGF- $\beta 1$ expression was observed. In addition, rats in the DM group exhibited glomerular enlargement with structural renal abnormalities, whilst those in the ME and HE groups showed improved symptoms. To conclude, no increased expression of
\end{abstract}

Correspondence to: Dr Yazhe Hu, Department of Sport Science, School of Physical Education, Central China Normal University, 152 Luoyu Road, Wuhan, Hubei 430070, P.R. China

E-mail: huyazhe@mail.ccnu.edu.cn

Key words: type 2 diabetes mellitus, endurance exercise, diabetic kidney damage, inflammatory cytokines inflammatory cytokines and renal fibrotic proteins could be observed in the present rat model of type-2 DM, but evident structural abnormalities can be observed in the kidneys. Medium-intensity endurance exercise can reduce serum inflammatory cytokine levels and prevent aberrant changes in renal structures.

\section{Introduction}

The global prevalence of diabetes has increased rapidly since the turn of the millennium (1). By 2025, the number of diabetic patients is predicted to reach 300 million in the world, with an increase rate of $200 \%$ in developing countries (1).

Diabetes mellitus (DM) is a metabolic disorder that is caused by high blood sugar levels as a result of either deficiencies in insulin secretion (type I) or insulin resistance (type II DM, or T2DM) $(2,3)$. Subsequently, utilization of glucose is hindered, which causes stress to multiple organs. Long term hyperglycemia can alter the metabolic and hemodynamic balance, in turn activating different inflammatory pathways and inducing injury to a number of organs, including blood vessels, heart, eyes, nerves and in particular the kidney (4). Diabetic nephropathy (DN) is a major complication that is associated with T2DM and also serve as the leading cause of end-stage renal disease (5). Renal microcirculation disorder and increased glycated protein levels can cause ischemia and stress damage to kidney tissues, which leads to glomerular injury (6).

DN is characterized by filtration barrier injury in the glomerulus, with features including glomerular mesangium expansion, increased glomerular basement membrane thickness and glomerulosclerosis, which is also known as glomerular atrophy (5). Transforming growth factor (TGF)- $\beta 1$ had been reported to be an important factor for DN pathogenesis that participates in the progression from glomerular sclerosis to interstitial fibrosis (7).

In addition, hyperglycemia can enhance TGF- $\beta 1$ expression, which stimulates the synthesis of extracellular matrix (ECM) proteins, including collagen, fibronectin and proteoglycan, whilst suppressing ECM degradation (8).

During latter stages of the disease, hyperglycemia can lead to atrophy and fibrosis of the glomerulus and DN progression (5).

The pathophysiology of DN remain to be fully elucidated. However, previous studies showed that in addition to 
hemodynamic and metabolic changes caused by hyperglycemia, activation of inflammatory pathways may also be an important underlying cause of DN $(5,9)$.

Chronic low-grade inflammation can induce $\mathrm{DN}$ in patients with T2DM (10). In particular, IL-6 was previously found to be a promising novel serum and urine marker for DN diagnosis and prognosis (11). Additonally, $\mathrm{CD}^{+}, \mathrm{CD}^{+} \mathrm{T}$ cell infiltration in the diabetic kidney has been demonstrated to increase the expression of IL- 6 and promote the development of DN in mice with streptozotocin (STZ)-induced diabetes $(12,13)$. Diet, medicine and exercise have traditionally been regarded to be the cornerstone of diabetes management (14). However, studies on the prevention and management of diabetes through exercise remain insufficient. Therefore, in the present study, rat T2DM models induced by high fat diet followed by injection with STZ were established, to study the effects of exercise intervention on the prevention of diabetes.

\section{Materials and methods}

Ethics approval. The present study was approved by the Ethics Committee of Central China Normal University (Wuhan, China).In total,50 specific pathogen-free male Sprague-Dawley rats (weight, 170-230 g; age, 6 weeks) were purchased from The Hubei Experimental Animal Research Center [license no. SCXK(E) 2015-0018, no. 42000600025097]. The animals were maintained on a 12-h light/dark cycle, at the temperature of $20-22^{\circ} \mathrm{C}$ with the relative humidity of $40-70 \%$ and allowed to eat and drink freely.

Animals and experimental design. After 1 week of adaptive feeding, rats were randomly divided into the following five groups ( $n=10$ rats per group): i) Control group (NC); ii) the diabetes group (DM); iii) the low-intensity exercise group (LE); iv) the moderate-intensity exercise group (ME); and v) the high-intensity exercise group (HE). One animal in the HE group died before the end of the experiment. At the same time of exercise intervention, rats in each group were fed with different diets. The NC group was fed with the national standard diet, whilst the DM, LE, ME and HE groups were all fed with high fat feed $(64.5 \%$ basic feed, $18 \%$ sucrose, $10 \%$ lard, $5 \%$ egg yolk powder, $2 \%$ cholesterol, $0.5 \%$ sodium bileate). After 8 weeks, rats were injected with STZ. A total of 1 week later, blood glucose was measured and the rats were euthanized.

Exercise regime. Rats in the NC and DM groups were allowed to move freely within their respective cages whereas those in the LE, ME and HE groups performed varying intensities of treadmill exercises during the feeding period with high fat diet. According to the preliminary experimental results of a pilot experiment and a previous study by Bedford et al (15), the exercise regimen designed for the present study was $8 \mathrm{~m} / \mathrm{min}$ and $0^{\circ}$ slope for the LE group, $15 \mathrm{~m} / \mathrm{min}$ and $5^{\circ}$ slope for the $\mathrm{ME}$ group and $20 \mathrm{~m} / \mathrm{min}$ and $10^{\circ}$ slope for the HE group. For $1 \mathrm{~h} /$ per day and 5 days per week, all three groups of rats that underwent exercise were able to complete $1 \mathrm{~h}$ of daily running training for 8 weeks. High-fat diet feeding were combined concomittantly with the three different intensity exercise regimens until the end of week 8 .
Animal model establishment. T2DM rat model was established according to protocols decribed in a previous study (16). Briefly, rats in all 5 groups were fasted overnight without water for $12 \mathrm{~h}$, whilst those in the NC group were given a single intraperitoneal injection of $0.1 \mathrm{mM}$ citrate buffer according to the dose of $30 \mathrm{mg} / \mathrm{kg} \mathrm{STZ}$. Rats in the DM, LE, ME and HE groups, in addition to undergoing exercise training for 8 weeks, were given a single intraperitoneal injection of $30 \mathrm{mg} / \mathrm{kg} 2 \%$ streptozotocin (STZ) solution after 8 weeks of HFD feeding. A total of 1 week later, $50 \mu \mathrm{l}$ blood collected from the tip of the rat's tail was used to measure blood glucose 1 week after STZ injection, where random blood glucose $\geq 16.7 \mathrm{mmol} / 1$ was used as the standard for T2MD modeling.

Oral Glucose tolerance test. Glucose solution was administered to all rats at a $50 \%$ concentration of glucose at a dose of $2 \mathrm{~g} / \mathrm{kg} 1$ week after STZ injection. A total of $200 \mu \mathrm{l}$ blood were collected directly from each rat's tail and blood glucose measurements were performed using a blood glucose meter before gavage and then 30,60 and $120 \mathrm{~min}$ after gavage, following which the blood glucose concentration was measured using a glucose meter (Sinacare). The trapezoidal method (17-18) was used to calculate the glucose area under the curve (AUC) during the OGTT. The formula is as follows: $\mathrm{AUC}_{\mathrm{FBG}}=$ Fasting blood glucose $(\mathrm{FBG})$ at $\left.0 \mathrm{~min}\right) \times 1 / 4+\mathrm{FBG}$ at $0 \mathrm{~min} \times 1 / 2+\mathrm{FBG}$ at $60 \mathrm{~min} \times 3 / 4+\mathrm{FBG}(120 \mathrm{~min}) \mathrm{x} 1 / 2$.

Measurement of serum biochemical indices. After anesthesia with $10 \%$ chloral hydrate $(400 \mathrm{mg} / \mathrm{kg}), 5 \mathrm{ml}$ blood was collected from the orbital sinus. The rats were then sacrificed by cervical dislocation. None of the rats showed any signs of peritonitis following administration of $10 \%$ chloral hydrate. Serum was separated by centrifugation at $2,000 \mathrm{x} g$ for $10 \mathrm{~min}$. ELISA was used to measure the protein levels in the serum. IL-6 (cat. no. EK0412) and TNF- $\alpha$ (cat. no. EK0526) was measured using ELISA kits (Boster Biological Technology) according to the manufacturer's protocols.The level of glycosylated serum protein (GSP) was measured using the GSP assay kit (cat. no A037-2, Nanjing Jiancheng Bioengineering Institute). Levels of blood urea nitrogen (BUN) and creatinine (Cr) were measured by an automatic biochemical analyzer (COBAS 701).

Western blot analysis. After euthanasia,the renal tissue was lysed using RIPA (Beyotime Institute of Biotechnology) on ice to extract the total protein. Protein concentration was determined using the bicinchoninic acid protein assay kit. A total of $40 \mu \mathrm{g}$ heat-denatured protein samples were separated by $10 \%$ SDS-PAGE. Subsequently, proteins were transferred onto PVDF membranes, followed by blocking with 5\% skimmed milk powder for $2 \mathrm{~h}$ at room temperature. The supernatant was collected as the total protein extract. The protein concentration was estimated by the Bradford Protein Assay kit (Aidlab Biotechnologies), according to the manufacturer's instructions. The membranes were then rinsed with TBS- $0.05 \%$ Tween-20 (TBST) and incubated with the following primary antibodiesat $4^{\circ} \mathrm{C}$ overnight: Mouse anti- $\beta$-actin antibody (cat. no. KM9001; Tiangen Sungene Biotech Co., Ltd.; 1:8,000), anti-matrix metalloproteinase (MMP) 9 (cat. no. 10375-2-AP; ProteinTech Group, Inc.; 1:1,000); anti-tissue inhibitors of metalloproteinases (TIMP)1 (cat. no. 10753-1-AP; ProteinTech group, Inc.; 
Table I. Changes in body weight of rats in the different groups at different stages.

\begin{tabular}{lcccc}
\hline Groups & $\mathrm{N}$ & Initial $(\mathrm{g})$ & End of week 8 $(\mathrm{g})$ & Day 7 after streptozotocin injection $(\mathrm{g})$ \\
\hline $\mathrm{NC}$ & 10 & $214.80 \pm 9.17$ & $496.56 \pm 36.01$ & $501.07 \pm 38.24$ \\
DM & 10 & $211.50 \pm 12.88$ & $549.86 \pm 15.08^{\mathrm{b}}$ & $499.80 \pm 47.99^{\mathrm{a}}$ \\
LE & 10 & $207.50 \pm 11.40$ & $520.23 \pm 43.36$ & $482.89 \pm 30.75^{\mathrm{a}}$ \\
ME & 10 & $212.00 \pm 13.28$ & $524.30 \pm 65.97$ & $511.53 \pm 86.00$ \\
HE & 9 & $202.4 \pm 30.52$ & $465.22 \pm 25.93^{\mathrm{c}-\mathrm{e}}$ & $432.00 \pm 19.32^{\mathrm{a}-\mathrm{e}}$ \\
\hline
\end{tabular}

Data are presented as the mean $\pm \mathrm{SD} .{ }^{\mathrm{a}} \mathrm{P}<0.05$ vs. end of the week 8 ; ${ }^{\mathrm{b}} \mathrm{P}<0.05$ vs. NC group; ${ }^{\mathrm{C}} \mathrm{P}<0.05$ vs. DM group; ${ }^{\mathrm{d}} \mathrm{P}<0.05$ vs. LE group; and ${ }^{\mathrm{e}} \mathrm{P}<0.05$ vs. ME group. NC, control group; DM, diabetes mellitus model group; LE, low intensity exercise intervention group; ME, moderate intensity exercise intervention group; HE, high intensity exercise intervention group.

1:1,000) and anti-TGF- $\beta 1$ (cat. no. 21898-1-AP; ProteinTech Group, Inc.; 1:1,000). After washing with TBST three times, PVDF membranes were incubuated overnight at $4{ }^{\circ} \mathrm{C}$ with the goat anti-rat secondary antibody (cat. no. ANT022; 1:10,000; Wuhan Antejie Biotechnology Co., Ltd.). After further washing with TBST, the membranes were submerged in BeyoECL Plus reagent (cat. no. P0018; Beyotime Institute of Biotechnology) and incubated for $1 \mathrm{~min}$ at room temperature. The membrane was wrapped in cling film and images were taken using a ChemiScope 3300 Mini gel imaging system (CLiNX; Scientific Instruments Co., Ltd.). The relative intensity of protein bands compared with those of $\beta$-actin was quantified using the Quantity One software (version 4.6.2; Bio-Rad Laboratories, Inc.).

Haematoxylin and eosin (H\&E) staining for histopathology. HE staining were performed to evaluate the renal pathological changes, glomerular morphological changes and renal fibrosis in the rats kidneys. Kidney tissues from all the rats were fixed with $4 \%$ paraformaldehyde at $4^{\circ} \mathrm{C}$ for $24 \mathrm{~h}$ and embedded in paraffin blocks before they were sectioned at $\sim 3-4 \mu \mathrm{m}$ thickness. The sections were treated with xylene for 5-10 min, immersed in a mixture of xylene and pure ethanol (1:1) for 5 min and then in 100, 95, 85 and $75 \%$ ethanol, each for $5 \mathrm{~min}$. Samples were then stained in Harris hematoxylin for $4 \mathrm{~min}$ at room temperature and rinsed in tap water for $2 \mathrm{~min}$. Differentiation was conducted using $1 \%$ hydrochloric acid, followed by washing with tap water. Slides were then counterstained with eosin for $2 \mathrm{~min}$ at room temperature. After eosin staining, the slices were dehydrated in 95 and $100 \%$ alcohol for $1 \mathrm{~min}$ at room temperature. The tissues were then stained with H\&E and evaluated at x400 magnification under a light microscope (Olympus BX51).

Glomerular diameter measurements. In total, six images of the glomerulus containing the vascular/urinary poles were randomly taken for each H\&E-stained kidney tissue section. NDP.view 2.6.13 image analysis software (Hamamatsu Photonics KK), was used to directly measure the maximum diameter of each glomerulus and to subsequently calculate the average glomerular diameter (19).

Statistical analysis. SPSS 20.0 (IBM Corp.) was used for statistics analysis and the data were expressed as the mean \pm SD.
Data were analyzed using one-way ANOVA with the Tukey's post hoc test. Mixed ANOVA followed by Bonferroni's test was used to measure differences in weight and fasting blood glucose (FBG) between groups at different time points. $\mathrm{P}<0.05$ was considered to indicate a statistically significant difference.

\section{Results}

Effects of exercise during DM modeling on the weight of rats. At the end of the 8th week, compared with those in the NC group, whilst rats in DM group showed significant body weight gain $(\mathrm{P}<0.05)$, no such increase was observed for rats in any of the three exercise groups. In addition, the weight of rats in the HE group was significantly lower compared with that in the LE and ME groups $(\mathrm{P}<0.05)$. Subsequently, 7 days after STZ injection, the weight of rats in HE group was lower compared with that in rats in the NC, DM, LE and ME groups $(\mathrm{P}<0.05)$. Compared with the weight of rats at the end of week 8 , those in rats in the DM, LE and $\mathrm{HE}$ groups were significantly reduced 1 week after STZ injection $(\mathrm{P}<0.05$; Table I).

Exercise can alleviate insulin resistance in rats with DM. No statistical difference was observed in the FBG levels among the groups after week 8. FBG levels remained unchanged 1 week after STZ injection in rats in the NC group but were increased significantly in those in the DM, LE, ME and HE groups $(\mathrm{P}<0.05)$ compared with week 8 . By contrast, FBG levels in rats in the ME and HE groups were significantly lower compared with those in the DM group $(\mathrm{P}<0.05)$. Additionally, GSP levels were measured 1 week after STZ injection. Compared with those in the NC, GSP levels were significantly increased in DM group $(\mathrm{P}<0.05)$. GSP levels were significantly decreased in $\mathrm{ME}$ group compared with those in the $\mathrm{DM}$ group $(\mathrm{P}<0.05$; Table II). However, there was no significant difference between other exercise groups and the DM group in terms of GSP.

The $\mathrm{AUC}_{\mathrm{FBG}}$ of the rats in $\mathrm{DM}$ group was significantly greater compared with that in the $\mathrm{NC}$ group $(\mathrm{P}<0.05)$, but there was no significant difference between that in $\mathrm{DM}$ and

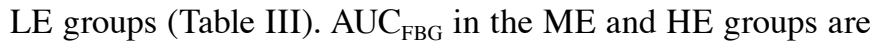
almost identical, but both are significantly lower compared with those in the DM group ( $\mathrm{P}<0.05$; Fig. 1; Table III).

Effect of exercise on serum inflammatory cytokine levels in each group. There were no significant differences between 
Table II. Fasting blood glucose and glycosylated serum protein levels.

\begin{tabular}{lcccc}
\hline & \multicolumn{4}{c}{ Fasting blood glucose $(\mathrm{mmol} / \mathrm{l})$} \\
\cline { 2 - 4 } Group & Week 1 & Week 8 & 1 week after streptozotocin injection & Glycated serum protein (mmol/l) \\
\hline NC & $4.91 \pm 0.56$ & $4.38 \pm 0.64$ & $4.87 \pm 0.50$ & $1.59 \pm 0.21$ \\
DM & $4.88 \pm 0.57$ & $4.65 \pm 0.57$ & $16.11 \pm 5.10^{\mathrm{a}, \mathrm{b}}$ & $2.92 \pm 0.85^{\mathrm{b}}$ \\
LE & $4.69 \pm 0.51$ & $4.29 \pm 0.68$ & $15.26 \pm 4.63^{\mathrm{a}, \mathrm{b}}$ & $2.48 \pm 0.42$ \\
ME & $4.79 \pm 0.59$ & $4.57 \pm 0.55$ & $9.09 \pm 3.40^{\mathrm{a}-\mathrm{d}}$ & $2.19 \pm 0.52^{\mathrm{c}}$ \\
HE & $4.72 \pm 0.65$ & $4.64 \pm 0.95$ & $9.00 \pm 3.88^{\mathrm{a}-\mathrm{d}}$ & $2.53 \pm 0.77$
\end{tabular}

Data are presented as the mean \pm SD. ${ }^{a} \mathrm{P}<0.05$ vs. Week $8 ;{ }^{\mathrm{b}} \mathrm{P}<0.05$ vs. $\mathrm{NC} ;{ }^{\mathrm{c}} \mathrm{P}<0.05$ vs. $\mathrm{DM}$; ${ }^{\mathrm{d}} \mathrm{P}<0.05$ vs. LE. NC, control group; DM, diabetes mellitus model group; LE, low intensity exercise intervention group; ME, moderate intensity exercise intervention group; HE, high intensity exercise intervention group.

Table III. $\mathrm{AUC}_{\mathrm{FBG}}$ on day 7 after injection of streptozotocin.

\begin{tabular}{lcl}
\hline Group & $\mathrm{N}$ & \multicolumn{1}{c}{$\mathrm{AUC}_{\mathrm{FBG}}$} \\
\hline $\mathrm{NC}$ & 10 & $13.73 \pm 0.95$ \\
$\mathrm{DM}$ & 10 & $47.18 \pm 10.20^{\mathrm{a}}$ \\
$\mathrm{LE}$ & 10 & $41.02 \pm 10.26$ \\
$\mathrm{ME}$ & 10 & $30.51 \pm 10.65^{\mathrm{b}}$ \\
$\mathrm{HE}$ & 9 & $30.52 \pm 13.68^{\mathrm{b}}$
\end{tabular}

Data are presented as the mean $\pm \mathrm{SD}$. ${ }^{\mathrm{a}} \mathrm{P}<0.05$ vs. $\mathrm{NC}$; ${ }^{\mathrm{b}} \mathrm{P}<0.05$ vs DM. NC, control group; DM, diabetes mellitus model group; LE, low intensity exercise intervention group; ME, moderate intensity exercise intervention group; HE, high intensity exercise intervention group; OGTT, oral glucose tolerance test; FBG, fasting blood glucose; AUC, area under the curve.

$\mathrm{NC}$ and DM in terms of both IL- 6 and TNF- $\alpha$. Compared with those in rats in the LE group, serum TNF- $\alpha$ levels were significantly lower in the ME and HE groups $(\mathrm{P}<0.05)$. Serum IL-6 levels were significantly lower in the LE and ME groups compared with those in the DM group $(\mathrm{P}<0.05$; Table IV). Although the difference of IL- 6 between DM and HE groups appeared to be large, there was no statistical difference.

Effects of exercise on renal function. Serum concentrations of urea and creatinine were subsequently measured 1 week after STZ injection, where no significant differences were observed among the five groups tested (Table V).

Effects of exercise on the expression MMP-9/TIMP-1 and TGF- $\beta 1$ in the kidney. The expression levels MMP-9, TIMP-1 and TGF- $\beta 1$ in renal tissue were next measured by western blotting 1 week after STZ injection. There were no statistically significant differences in the expression levels of MMP-9, TIMP-1 and TGF- $\beta 1$ in tissues among the five groups tested (Fig. 2).

Effects of exercise on the kidney morphological structure and glomerular diameter. Compared with that in the $\mathrm{NC}$ group, glomerular diameter exhibited a significant increase in DM and LE groups $(\mathrm{P}<0.05)$. Compared with that in the DM group,

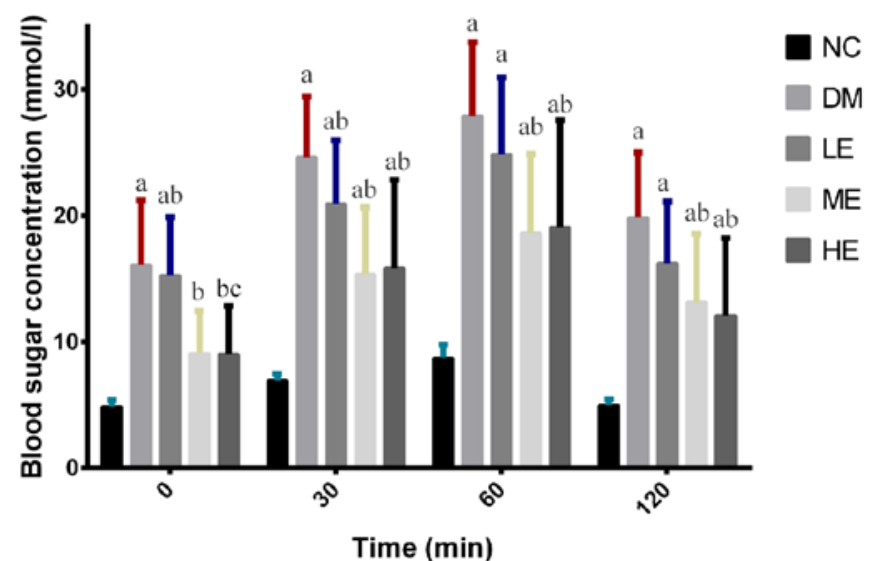

Figure 1. Serum glucose levels in oral glucose tolerance test in each group of rats. After feeding with glucose in rats orally, blood glucose levels were measured at 0,30, 60, 90 and $120 \mathrm{~min}$ and were under the oral glucose tolerance test blood glucose curve. Data are presented as the mean $\pm \mathrm{SD}$. ${ }^{\mathrm{a}} \mathrm{P}<0.05$ vs. NC, ${ }^{\mathrm{b}} \mathrm{P}<0.05$ vs. $\mathrm{DM},{ }^{\mathrm{C}} \mathrm{P}<0.05$ vs. LE. NC, control group; $\mathrm{DM}$, diabetes mellitus model group; LE, low intensity exercise intervention group; $\mathrm{ME}$, moderate intensity exercise intervention group; $\mathrm{HE}$, high intensity exercise intervention group.

glomerular diameter was significantly reduced in the ME and HE groups $(\mathrm{P}<0.05)($ Table VI).

No notable pathological changes were observed in the kidney slices obtained from rats in the NC group by H\&E staining. In the tissues from the DM group, histological analysis revealed unclear glomerular structures, glomerular hypertrophy, increased basal membrane thickness and mesangial region width, in addition to increased glomerular cell number. In the kidney tissues of rats in the LE group, glomerular hypertrophy was also observed, along with increased glomerular cell number and slight increases in the mesangial region width. The renal tubular and glomerular damage in the $\mathrm{ME}$ and HE groups were notably reduced, where the number of glomerular cells were also reduced without evident glomerular hypertrophy (Fig. 3).

\section{Discussion}

In the present study, T2DM rat model was established by adopting high fat diet in combination with STZ injection, 
Table IV. Serum inflammatory factor content in each group.

\begin{tabular}{lccc}
\hline Group & $\mathrm{N}$ & TNF- $\alpha(\mathrm{pg} / \mathrm{ml})$ & IL-6 $(\mathrm{pg} / \mathrm{ml})$ \\
\hline $\mathrm{NC}$ & 10 & $8.88 \pm 4.22$ & $74.66 \pm 41.06$ \\
DM & 10 & $9.00 \pm 4.35$ & $88.37 \pm 48.63$ \\
LE & 10 & $11.71 \pm 5.80$ & $30.79 \pm 18.56^{\mathrm{a}}$ \\
ME & 10 & $5.14 \pm 3.72^{\mathrm{a}, \mathrm{b}}$ & $31.64 \pm 19.73^{\mathrm{a}}$ \\
HE & 9 & $2.85 \pm 1.22^{\mathrm{a}, \mathrm{b}}$ & $51.77 \pm 29.80$ \\
\hline
\end{tabular}

Data are presented as the mean $\pm \mathrm{SD}$. ${ }^{\mathrm{a}} \mathrm{P}<0.05$ vs. $\mathrm{DM}$; ${ }^{\mathrm{b}} \mathrm{P}<0.05$ vs. LE. NC, control group; DM, diabetes mellitus model group; LE, low intensity exercise intervention group; $\mathrm{ME}$, moderate intensity exercise intervention group; HE, high intensity exercise intervention group; TNF, tumor necrosis factor; IL, interleukin.

which exhibits similar pathophysiology to that of T2DM caused by obesity in humans (20).

This method confers many advantages, including short experiment duration, ease of establishment, low cost and relative reliability, rendering it to be one of the most commonly applied methods for modeling T2DM $(21,22)$. Using this method, insulin resistance was induced in animals by feeding them with high fat diet, followed by injection with low doses of STZ, which enters the $\beta$ cells in the pancreas via the GLUT2 transporters (23).

In the $\beta$ cells, STZ is hydrolyzed into glucose and methyl nitrosourea, with the latter causing DNA break and cytotoxicity $(24,25)$.

Subsequent $\beta$ cell damage leads to the failure of insulin synthesis and release by the pancreas, which in return causes increases in blood sugar levels. The characteristics of this model were insulin resistance but normal plasma insulin levels, middle to high level blood sugar levels and high blood lipid levels $(21,22)$.

The precise pathogenesis of DN remain unclear. However, it has been reported that hyperglycemia promotes renal inflammation, oxidative stress and renal fibrosis, all of which serve important roles in the development of this condition $(26,27)$.

Although no difference in the FBG levels was observed among the all groups at 8 weeks, FBG levels were significantly lower in the ME and HE groups compared with those in the LE and DM groups 1 week after STZ injection. This suggests that moderate to high-intensity endurance exercise helps to suppress the elevation of blood sugar levels induced by DM. This observation was also in line with glycosylated serum protein levels which was measured in the present study. GSP level is a good indicator to reflect the average level of blood glucose in the prior 1-2 weeks (28). In the present experiment, due to STZ injection in the last week, in order to better evaluate the change of blood glucose, GSP was used for auxiliary evaluation.

Patients with impaired glucose tolerance patient frequently exhibit periphery insulin resistance, impaired glucose metabolism in the muscle and adipose tissues, coupled with deficiency in insulin secretion $(29,30)$.

In the present study, it was demonstrated that exercise intervention during the model construction phase can reduce
Table V. Concentration of serum urea and creatinine.

\begin{tabular}{lrcc}
\hline Group & $\mathrm{N}$ & Urea $(\mathrm{mmol} / \mathrm{l})$ & Creatinine $(\mu \mathrm{mol} / \mathrm{l})$ \\
\hline $\mathrm{NC}$ & 10 & $1.13 \pm 0.15$ & $6.00 \pm 1.25$ \\
$\mathrm{DM}$ & 10 & $1.09 \pm 0.14$ & $5.80 \pm 0.63$ \\
$\mathrm{LE}$ & 10 & $1.24 \pm 0.24$ & $5.44 \pm 0.88$ \\
$\mathrm{ME}$ & 10 & $1.00 \pm 0.23$ & $6.30 \pm 1.49$ \\
$\mathrm{HE}$ & 9 & $1.16 \pm 0.22$ & $5.56 \pm 0.88$ \\
\hline
\end{tabular}

Data are presented as the mean $\pm \mathrm{SD}$. NC, control group; DM, diabetes mellitus model group; LE, low intensity exercise intervention group; $\mathrm{ME}$, moderate intensity exercise intervention group; HE, high intensity exercise intervention group.

the severity of impaired glucose tolerance. The $\mathrm{AUC}_{\mathrm{FBG}}$ values in theME and $\mathrm{HE}$ groups were significantly lower compared with those in the DM group. Furthermore, compared with that in the $\mathrm{LE}$ group, the lower $\mathrm{AUC}_{\mathrm{FBG}}$ values in the $\mathrm{ME}$ and $\mathrm{HE}$ groups indicates that middle and high intensity endurance exercises are more efficient at improving glucose metabolism by peripheral tissues. Therefore, moderate-intensity exercise during the pre-diabetes period can potentially prevent impairments in sugar metabolism.

T2DM is a chronic inflammatory condition caused by adipose cell dysfunction induced by obesity (31). Adipose cells can produce excessive amounts of inflammatory cytokines, including TNF- $\alpha$ and IL-6 (32). This chronic inflammatory state further promotes insulin resistance $(33,34)$. Patients with DN typically display significantly elevated serum and urine levels of inflammatory cytokines, which has been previously demonstrated to correlate with the progression of $\mathrm{DN}(35,36)$. Belotto et al (37) reported that 3 weeks of exercise can reduce the level of serum TNF- $\alpha$, IL-6 in rats with DM, where middle-intensity exercise exerted notable anti-inflammatory effects in rats with DM rats. In the present study, all three exercise regimens were demonstrated to reduce TNF- $\alpha$ and IL-6 cytokine levels in the serum by different degrees (compared to that in the DM group, TNF- $\alpha$ in the ME and HE groups was decreased and IL-6 in the LE and ME groups was decreased), with effects mediated by moderate-intensity exercise being superior compared with those by low and high-intensity exercises. This finding was consistent with that reported by Belotto et al (37).

The mechanism of DN pathogenesis include hemodynamic changes in the kidney induced by hyperglycemia, non-enzymatic protein glycosylation, and the aberrant sorbitol metabolism of cellular glucose (38).

All of those aforementioned can result in the elevation and activation of IL- 6 , and TNF- $\alpha$ causing lesions in the kidney tubules and increases in extracellular matrix deposition in the glomerular mesangial matrix (39). Fakhruddin S, Alanazi W and Jackson KE: Diabetes-induced reactive oxygen species: Mechanism of their generation and role in renal injury. Journal of diabetes research 2017: 2017. Diabetic damage to the kidney is progressive, with its early histological manifestation characterized by glomerular hypertrophy, followed by the increases in GBM thickness, excessive accumulation of collagen 

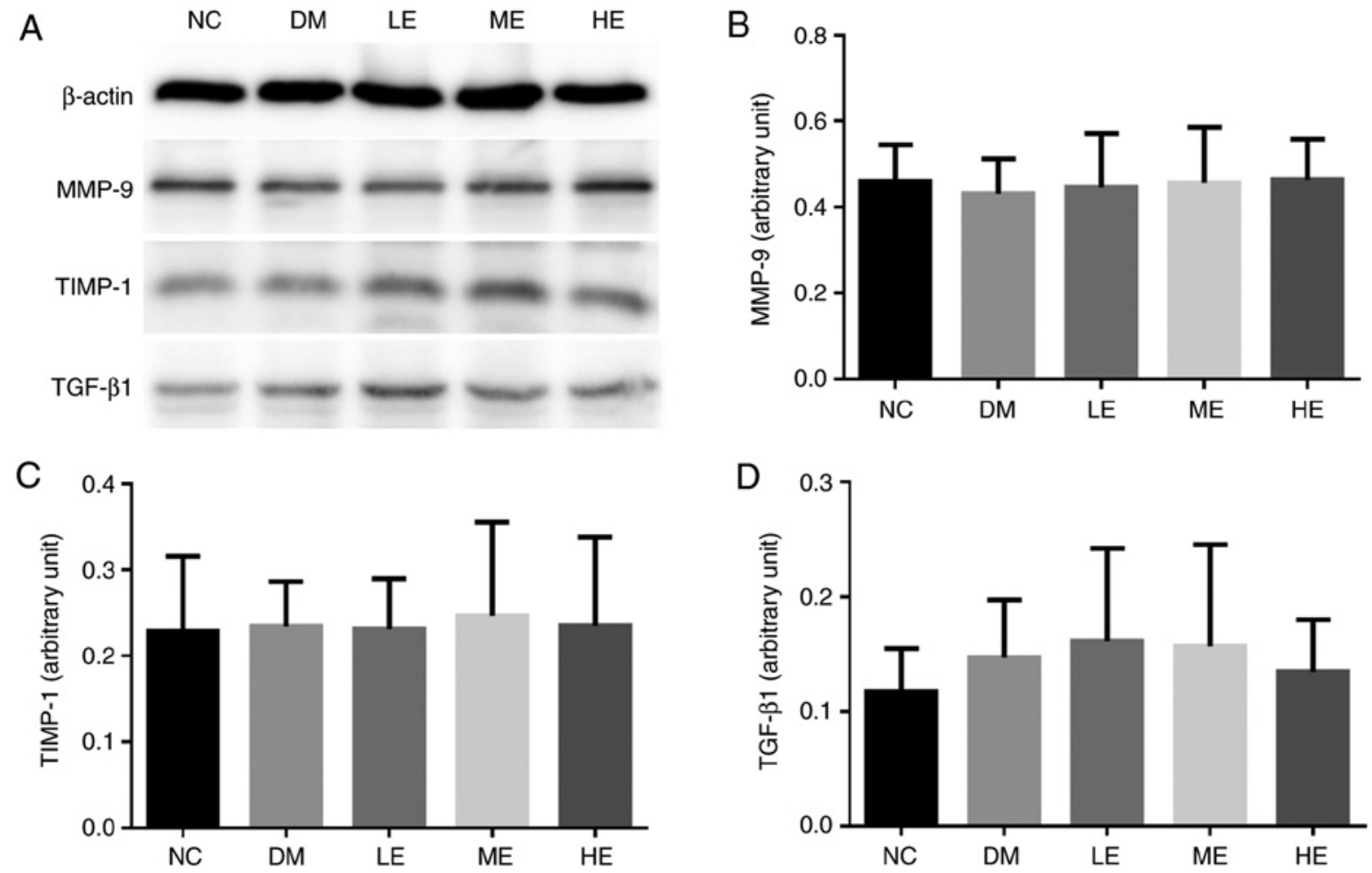

Figure 2. Expression of MMP-9/TIMP-1 protein in the kidney tissues from rats in each group. (A) Representative western blotting images of the expression of MMP-9, TIMP-1 and TGF- $\beta 1$. Quantification of (B) MMP-9, (C) TIMP-1 and (D) TGF- $\beta 1$. Data represent the mean \pm SD ( $\mathrm{n}=10$ ). NC, control group; DM, diabetes mellitus model group; LE, low intensity exercise intervention group; ME, moderate intensity exercise intervention group; HE, high intensity exercise intervention group; MMP, matrix metalloproteinase; TIMP, tissue inhibitors of metalloproteinase; TGF, transforming growth factor.

Table VI. Glomerular diameter measurements.

\begin{tabular}{lcc}
\hline Group & $\mathrm{N}$ & Diameter $(\mu \mathrm{m})$ \\
\hline $\mathrm{NC}$ & 10 & $138.22 \pm 5.23$ \\
$\mathrm{DM}$ & 10 & $155.43 \pm 4.20^{\mathrm{a}}$ \\
$\mathrm{LE}$ & 10 & $157.67 \pm 3.69^{\mathrm{a}}$ \\
$\mathrm{ME}$ & 10 & $137.75 \pm 2.23^{\mathrm{b}}$ \\
$\mathrm{HE}$ & 9 & $135.22 \pm 3.63^{\mathrm{b}}$ \\
\hline
\end{tabular}

Data are presented as the mean $\pm \mathrm{SD} .{ }^{\mathrm{a}} \mathrm{P}<0.05$ vs. $\mathrm{NC}$; ${ }^{\text {b }} \mathrm{P}<0.05$ vs DM. NC, control group; DM, diabetes mellitus model group; LE, low intensity exercise intervention group; ME, moderate intensity exercise intervention group; HE, high intensity exercise intervention group.

type IV, sedimentation of mesangial matrix and increases in the excretion ratio of urinary proteins, which finally results in glomerulosclerosis and renal failure $(39,40)$.

TGF- $\beta 1$ can stimulate the expression of monocyte chemoattractant protein-1 in mesangial cells (35), which is known to be an important mediator in ECM and collagen deposition and fibrosis formation during DN (41). Kuusniemi et al (41) hypothesized that renal tubular epithelial cells can directly promote fibrosis by ECM protein generation. The accumulation of ECM proteins was found to be the result of increases in synthesis, reductions in degradation or the combination of both. Under physiological conditions, synthesis and degradation of the ECM is maintained in a dynamic balance (42). MMPs and TIMPs are the main mediators of ECM degradation (43). In particular, MMP-9 is an important member of the MMPs family that is able to degrade multiple types of ECM proteins, such as type IV collagen and endothelial junctional protein $(44,45)$.

Type IV collagen mainly exists in the kidney basal membrane, where its catabolism is generally slow unless specific degradative enzymes are activated (46). The combination of MMP-9 and TIMP-1 activities can keep the degradation and resynthesis of type IV collagen in balance (44). In the present study, the expression levels of MMP-9 and TIMP-1 in the kidney did not vary among the five experimental groups, suggesting that during the early stages of T2DM, ECM synthesis and degradation in the rat kidney tissue was still maintained in a dynamic balance, where this balance between MMP-9 and TIMP-1 was not disturbed. This was also supported by the comparable expression levels of kidney TGF- $\beta 1$ expression among these groups. Therefore, it is reasonable to suggest that at the onset of diabetes, overexpression of MMP-9 and TGF- $\beta 1$ may not be evident even though sugar levelswere disturbed.

In the present study, evident pathological changes were observed in the kidneys of rats in the DM group, which were prevented by endurance exercises. In addition, the present study also measured the glomerular diameter to evaluate the degree of glomerular hypertrophy. There was an increase in glomerular diameter and glomerular hypertrophy during the early stages of T2DM, which could be prevented by endurance exercise of moderate and high intensities. 


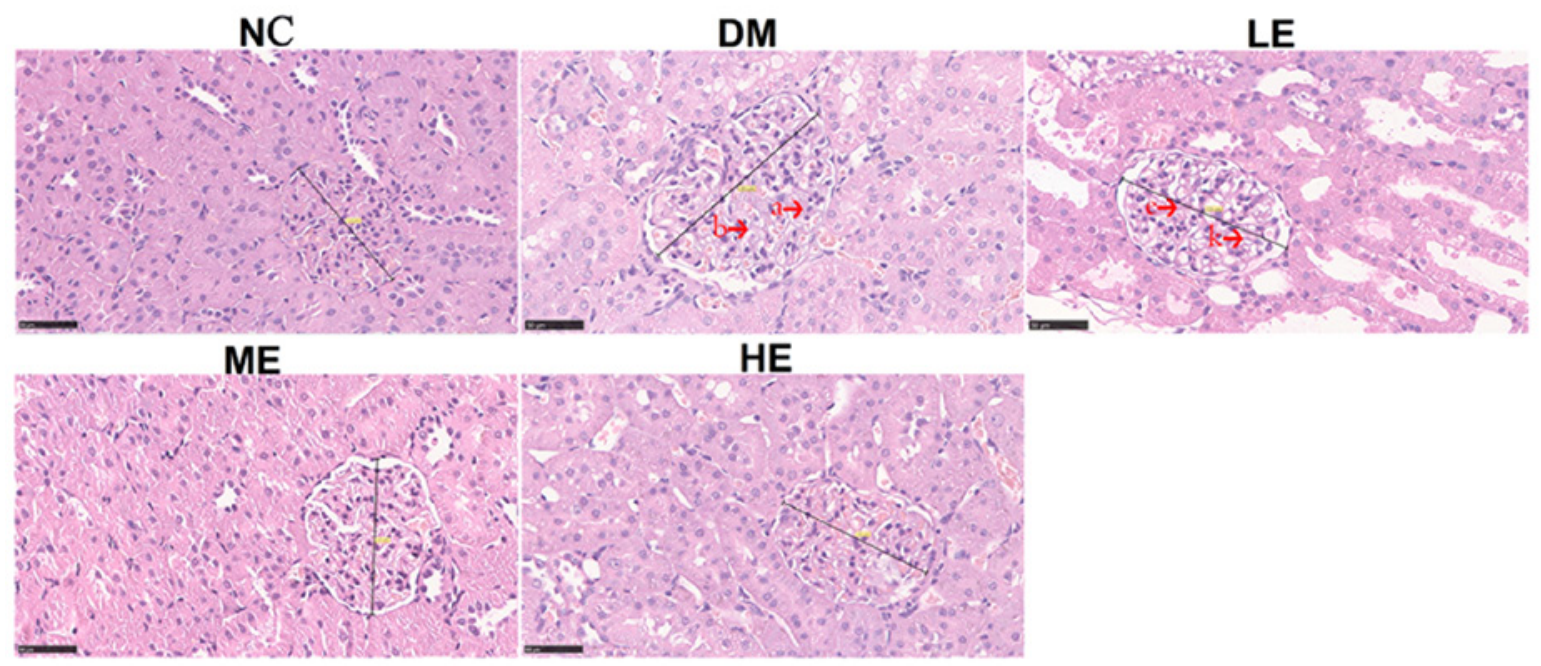

Figure 3. H\&E staining results of the kidneys from rats in each group. In comparison to NC group, the glomerular basement membrane is thickened in the DM and LE groups (arrows b and k). In addition, the mesangial area is thickened in the DM and LE group (arrows a and e). Diameters of glomeruli were measured. In group DM and LE, glomerular diameter increased. Scale bar, $50 \mu \mathrm{m}$. NC, control group; DM, diabetes mellitus model group; LE, low intensity exercise intervention group; ME, moderate intensity exercise intervention group; HE, high intensity exercise intervention group.

Eddy (47) previously divided the pathological process of renal tubulointerstitial fibrosis into four stages. The first of which is cell activation and damage stage, where the peritubular capillary endothelium can promote the migration of monocytes to the renal interstitium where they would mature to become macrophages. At this stage, myofibroblasts and activated fibroblasts would start filling the interstitium., where soluble products, including fibronectin and alarmins, released from these cells would cause persistent inflammation (47). The levels of inflammatory cytokines TNF- $\alpha$ and IL-6 were not found to be altered in the DM group compared to that in the NC group in the present study. This suggests that the renal fibrosis process did not begin one week after the end of modeling.

The second stage of fibrosis is known as the fibrotic signaling stage (47). At this stage, a variety of growth factors and cytokines are involved, where factors, such as TGF- $\beta$ serve a pivotal role. Excessive secretion of TGF- $\beta 1$ has been widely reported to be one of the major molecular events of fibrotic diseases (27). Other factors, including blood TNFs and ILs, may also be involved (26). In the third stage, matrix protein synthesis is increased where matrix transformation becomes impaired, in manner that is caused by increases in the expression of factors such as TIMPs. In the present study, expression levels of neither TGF- $\beta 1$ nor MMPs in DM group exhibited significant difference among the five groups. The fourth stage is known as the kidney damage stage (47), which constitutes as the ultimate consequence of excessive matrix accumulation.

All of the effects aforementioned suggest further that renal tubule interstitial fibrosis may not occur during the early stages of diabetes. Although rats in the DM groups showed no differences in the inflammatory cytokine levels, endurance exercises of varying intensities reduced the inflammatory cytokine levels in the body to various degrees. Although a number of studies previously found that exercise can reduce inflammatory cytokine levels $(48,49)$, few have reported the effects of exercise intervention during the process of T2DM induction. The results of the present study suggest that exercise may inhibit renal fibrosis by reducing the inflammation level in the body, thereby protecting the renal structure from further damage. However, a limitation of the present study was that all experiments were terminated 1 week after STZ injection, at which time the rats could still be at the early stages of T2DM, such that the extent of kidney damage may not have been significant, since most indicators did not show differences.

In conclusion, at the onset of T2DM, whilst the levels of inflammatory cytokines were not significantly increased, disrupted renal structures and the compensatory glomerulus hypertrophy were observed. Exercise intervention could reduce the inflammation levels and inhibit renal fibrosis by improving glucose metabolism, which served to preserve renal structure and function.

\section{Acknowledgements}

Not applicable.

\section{Funding}

No funding was received.

\section{Availability of data and materials}

The datasets used and/or analyzed during the current study are available from the corresponding author on reasonable request.

\section{Authors' contributions}

SL performed animal experiments and immunohistochemical staining. SJ and MZ performed molecular biology experiments. FZ analyzed the data. YH designed the present study and prepared the manuscript. SL and MZ revised the manuscript. All authors read and approved the final manuscript. 


\section{Ethics approval and consent to participate}

All of the animal procedures were approved by the Animal Care and Use Committee at Central China Normal University (Wuhan, China).

\section{Patient consent for publication}

Not applicable.

\section{Competing interests}

The authors declare that they have no competing interests.

\section{References}

1. Green A, Christian Hirsch N and Pramming SK: The changing world demography of type 2 diabetes. Diabetes Metab Res Rev 19: 3-7, 2003.

2. Zimmet P: Globalization, coca-colonization and the chronic disease epidemic: Can the Doomsday scenario be averted? J Intern Med 247: 301-310, 2000.

3. Zimmet P, Alberti KG and Shaw J: Global and societal implications of the diabetes epidemic. Nature 414: 782-787, 2001.

4. Rask-Madsen C and King GL: Vascular complications of diabetes: Mechanisms of injury and protective factors. Cell Metab 17: 20-33, 2013.

5. Piccoli GB, Grassi G, Cabiddu G, Nazha M, Roggero S, Capizzi I, De Pascale A, Priola AM, Di Vico C, Maxia S, et al: Diabetic kidney disease: A syndrome rather than a single disease. Rev Diabet Stud 12: 87-109, 2015.

6. Jha JC, Banal C, Chow BS, Cooper ME and Jandeleit-Dahm K: Diabetes and kidney disease: Role of oxidative stress. Antioxid Redox Signal 25: 657-684, 2016.

7. Huang K, Chen C, Hao J, Huang J, Wang S, Liu P and Huang H: Polydatin promotes Nrf2-ARE anti-oxidative pathway through activating Sirt1 to resist AGEs-induced upregulation of fibronetin and transforming growth factor- $\beta 1$ in rat glomerular messangial cells. Mol Cell Endocrinol 399: 178-189, 2015.

8. Hu C, Sun L, Xiao L, Han Y, Fu X, Xiong X, Xu X, Liu Y, Yang S, Liu F and Kanwar YS: Insights into the mechanisms involved in the expression and regulation of extracellular matrix proteins in diabetic nephropathy. Curr Med Chem 22: 2858-2870, 2015.

9. Duran-Salgado MB and Rubio-Guerra AF: Diabetic nephropathy and inflammation. World J Diabetes 5: 393-398, 2014.

10. Yeo ES, Hwang JY, Park JE, Choi YJ, Huh KB and Kim WY: Tumor necrosis factor (TNF-alpha) and C-reactive protein (CRP) are positively associated with the risk of chronic kidney disease in patients with type 2 diabetes. Yonsei Med J 51: 519-525, 2010.

11. Campion CG, Sanchez-Ferras O and Batchu SN: Potential role of serum and urinary biomarkers in diagnosis and prognosis of diabetic nephropathy. Can J Kidney Health Dis 4: $2054358117705371,2017$.

12. Dalla Vestra M, Mussap M, Gallina P, Bruseghin M, Cernigoi AM, Saller A, Plebani M and Fioretto P: Acute-phase markers of inflammation and glomerular structure in patients with type 2 diabetes. J Am Soc Nephrol 16 (Suppl 1): S78-S82, 2005.

13. Moon JY, Jeong KH, Lee TW, Ihm CG, Lim SJ and Lee SH: Aberrant recruitment and activation of $\mathrm{T}$ cells in diabetic nephropathy. Am J Nephrol 35: 164-174, 2012.

14. Sigal RJ, Kenny GP, Wasserman DH and Castaneda-Sceppa C: Physical activity/exercise and type 2 diabetes. Diabetes Care 27: 2518-2539, 2004.

15. Bedford TG, Tipton CM, Wilson NC, Oppliger RA and Gisolfi CV: Maximum oxygen consumption of rats and its changes with various experimental procedures. J Appl Physiol Respir Environ Exerc Physiol 47: 1278-1283, 1979.

16. Guo XX, Wang Y, Wang K, Ji BP and Zhou F: Stability of a type 2 diabetes rat model induced by high-fat diet feeding with low-dose streptozotocin injection. J Zhejiang Univ Sci B 19: $559-569,2018$
17. Stančáková A, Kuulasmaa T, Paananen J, Jackson AU, Bonnycastle LL, Collins FS, Boehnke M, Kuusisto J and Laakso M: Association of 18 confirmed susceptibility loci for type 2 diabetes with indices of insulin release, proinsulin conversion, and insulin sensitivity in 5,327 nondiabetic Finnish men. Diabetes 58: 2129-2136, 2009.

18. Cantrell Stanford J, Morris AJ, Sunkara M, Popa GJ, Larson KL and Özcan S: Sphingosine 1-phosphate (S1P) regulates glucose-stimulated insulin secretion in pancreatic beta cells. J Biol Chem 287: 13457-13464, 2012.

19. Cheng H, Dong HR, Lin RQ, Sun LJ and Chen YP: Determination of normal value of glomerular size in Chinese adults by different measurement methods. Nephrology (Carlton) 17: 488-492, 2012.

20. Nath S, Ghosh SK and Choudhury Y: A murine model of type 2 diabetes mellitus developed using a combination of high fat diet and multiple low doses of streptozotocin treatment mimics the metabolic characteristics of type 2 diabetes mellitus in humans. J Pharmacol Toxicol Methods 84: 20-30, 2017.

21. Chatzigeorgiou A, Halapas A, Kalafatakis $\mathrm{K}$ and Kamper E: The use of animal models in the study of diabetes mellitus. In Vivo 23: 245-258, 2009.

22. Eleazu CO, Eleazu KC, Chukwuma S and Essien UN: Review of the mechanism of cell death resulting from streptozotocin challenge in experimental animals, its practical use and potential risk to humans. J Diabetes Metab Disord 12: 60, 2013.

23. Elsner M, Guldbakke B, Tiedge M, Munday R and Lenzen S: Relative importance of transport and alkylation for pancreatic beta-cell toxicity of streptozotocin. Diabetologia 43: 1528-1533, 2000.

24. Mohamed AH, Abd El-Hameed MN, Khamis HH, Sharkawy GK and Samir AE: Antidiabetic and anti-inflammatory effects of two Fabaceae extracts against streptozotocin induced diabetic impairment in male rats. World J Adv Res Rev 6: 12-29, 2020.

25. Bennett RA and Pegg AE: Alkylation of DNA in rat tissues following administration of streptozotocin. Cancer Res 41: 2786-2790, 1981

26. Turkmen K: Inflammation, oxidative stress, apoptosis, and autophagy in diabetes mellitus and diabetic kidney disease: The four Horsemen of the apocalypse. Int Urol Nephrol 49: 837-844, 2017.

27. Sharma K: Obesity, oxidative stress, and fibrosis in chronic kidney disease. Kidney Int Suppl (2011) 4: 113-117, 2014.

28. Goldstein DE, Little RR, Lorenz RA, Malone JI, Nathan D, Peterson CM and Sacks DB: Tests of glycemia in diabetes. Diabetes Care 27: 1761-1773, 2004.

29. DeFronzo RA and Tripathy D: Skeletal muscle insulin resistance is the primary defect in type 2 diabetes. Diabetes Care 32 (Suppl 2): S157-S163, 2009.

30. Muoio DM and Newgard CB: Mechanisms in disease: Molecular and metabolic mechanisms of insulin resistance and beta-cell failure in type 2 diabetes. Nat Rev Mol Cell Biol 9: 193-205, 2008.

31. Donath MY and Shoelson SE: Type 2 diabetes as an inflammatory disease. Nat Rev Immunol 11: 98-107, 2011.

32. Zinman B, Hanley AJ, Harris SB, Kwan J and Fantus IG: Circulating tumor necrosis factor- $\alpha$ concentrations in a native Canadian population with high rates of type 2 diabetes mellitus. J Clin Endocrinol Metab 84: 272-278, 1999.

33. Pradhan AD, Manson JE, Rifai N, Buring JE and Ridker PM: C-reactive protein, interleukin 6 , and risk of developing type 2 diabetes mellitus. JAMA 286: 327-334, 2001.

34. Abrahamian H, Endler G, Exner M, Mauler H, Raith M, Endler L, Rumpold H, Gerdov M, Mannhalter C, Prager R, et al: Association of low-grade inflammation with nephropathy in type 2 diabetic patients: Role of elevated CRP-levels and 2 different gene-polymorphisms of proinflammatory cytokines. Exp Clin Endocrinol Diabetes 115: 38-41, 2007.

35. Soetikno V, Sari FR, Veeraveedu PT, Thandavarayan RA, Harima M, Sukumaran V, Lakshmanan AP, Suzuki K, Kawachi $\mathrm{H}$ and Watanabe K: Curcumin ameliorates macrophage infiltration by inhibiting NF- $\mathrm{BB}$ activation and proinflammatory cytokines in streptozotocin induced-diabetic nephropathy. Nutr Metab (Lond) 8: 35, 2011.

36. Wolkow PP, Niewczas MA, Perkins B, Ficociello LH, Lipinski B, Warram JH and Krolewski AS: Association of urinary inflammatory markers and renal decline in microalbuminuric type 1 diabetics. J Am Soc Nephrol 19: 789-797, 2008.

37. Belotto MF, Magdalon J, Rodrigues HG, Vinolo MA, Curi R, Pithon-Curi TC and Hatanaka E: Moderate exercise improves leucocyte function and decreases inflammation in diabetes. Clin Exp Immunol 162: 237-243, 2010. 
38. Kumar Pasupulati A, Chitra PS and Reddy GB: Advanced glycation end products mediated cellular and molecular events in the pathology of diabetic nephropathy. Biomol Concepts 7: 293-309, 2016.

39. Fakhruddin S, Alanazi W and Jackson KE: Diabetes-induced reactive oxygen species: Mechanism of their generation and role in renal injury. J Diabetes Res 2017: 8379327, 2017.

40. Diez-Marques L, Ortega-Velazquez R, Langa C, RodriguezBarbero A, Lopez-Novoa JM, Lamas S and Bernabeu C: Expression of endoglin in human mesangial cells: Modulation of extracellular matrix synthesis. Biochim Biophys Acta 1587: 36-44, 2002.

41. Kuusniemi AM, Lapatto R, Holmberg C, Karikoski R, Rapola J and Jalanko H: Kidneys with heavy proteinuria show fibrosis, inflammation, and oxidative stress, but no tubular phenotypic change. Kidney Int 68: 121-132, 2005.

42. Lu P, Takai K, Weaver VM and Werb Z: Extracellular matrix degradation and remodeling in development and disease. Cold Spring Harb Perspect Biol 3: a005058, 2011.

43. Tan RJ and Liu Y: Matrix metalloproteinases in kidney homeostasis and diseases. Am J Physiol Renal Physiol 302: F1351-F1361, 2012.

44. Catania JM, Chen G and Parrish AR: Role of matrix metalloproteinases in renal pathophysiologies. Am J Physiol Renal Physiol 292: F905-F911, 2007.
45. Song L, Ge S and Pachter JS: Caveolin-1 regulates expression of junction-associated proteins in brain microvascular endothelial cells. Blood 109: 1515-1523, 2007.

46. Mariappan MM: Signaling mechanisms in the regulation of renal matrix metabolism in diabetes. Exp Diabetes Res 2012: 749812, 2012.

47. Eddy AA: Molecular basis of renal fibrosis. Pediatr Nephrol 15: 290-301, 2000

48. Abd El-Kader SM: Aerobic versus resistance exercise training in modulation of insulin resistance, adipocytokines and inflammatory cytokine levels in obese type 2 diabetic patients. J Adv Res 2: 179-183, 2011.

49. Stewart LK, Flynn MG, Campbell WW, Craig BA, Robinson JP, Timmerman KL, McFarlin BK, Coen PM and Talbert E: The influence of exercise training on inflammatory cytokines and C-reactive protein. Med Sci Sports Exerc 39: 1714-1719, 2007.

This work is licensed under a Creative Commons Attribution-NonCommercial-NoDerivatives 4.0 International (CC BY-NC-ND 4.0) License. 